\title{
Auditory and Visual User Interface for Optical Guidance During Stereotactic Brain Tumor Biopsies
}

Michaela Maintz, David Black and Neda Haj-Hosseini

The self-archived postprint version of this journal article is available at Linköping University Institutional Repository (DiVA):

http:/ / urn.kb.se/ resolve?urn=urn:nbn:se:liu:diva-161068

N.B.: When citing this work, cite the original publication.

Maintz, M., Black, D., Haj-Hosseini, N., (2019), Auditory and Visual User Interface for Optical Guidance During Stereotactic Brain Tumor Biopsies, 2019 41ST ANNUAL INTERNATIONAL CONFERENCE OF THE IEEE ENGINEERING IN MEDICINE AND BIOLOGY SOCIETY (EMBC), , 1981-1984. https:// doi.org/ 10.1109/ EMBC.2019.8857310

Original publication available at:

https:/ / doi.org/ 10.1109/ EMBC.2019.8857310

Copyright: Institute of Electrical and Electronics Engineers (IEEE)

http:// www.ieee.org/

(C) 2019 IEEE. Personal use of this material is permitted. However, permission to reprint/ republish this material for advertising or promotional purposes or for creating new collective works for resale or redistribution to servers or lists, or to reuse any copyrighted component of this work in other works must be obtained from the IEEE. 


\title{
Auditory and Visual User Interface for Optical Guidance During Stereotactic Brain Tumor Biopsies
}

\author{
Michaela Maintz ${ }^{1,2}$, David Black ${ }^{3}$ and Neda Haj-Hosseini ${ }^{1}$
}

\begin{abstract}
During stereotactic brain tumor biopsies, the detection of protoporphyrin IX (PpIX) fluorescence and microvascular perfusion using laser Doppler flowmetry (LDF) with a handheld fiber optic probe allows the identification of tumor tissue while decreasing the risk of intracranial hemorrhage. Neurosurgeons performing this procedure usually view the measurement values on a screen. When their visual focus is directed at the surgical site, they require an assistant to verbally relay the values. An auditory and visual user interface (UI), which displays measurement values accurately and allows fast and intuitive signal recognition, can improve this procedure. This paper experimentally evaluates an auditory and visual UI for providing real-time measurement feedback during stereotactic brain tumor biopsies. In a user study $(n=15)$, the accuracy of auditory and visual response was determined using function response tests, and user acceptance was evaluated. The auditory signals proved to be intuitive and easy to recognize and remember. The visual display of measurement values was easy to understand and facilitated the user's decision-making process. Moreover, the UI exhibited high user acceptance.
\end{abstract}

\section{INTRODUCTION}

In stereotactic brain tumor biopsy procedures, tissue samples are extracted for the diagnosis of tumors from brain areas that are difficult to access. Preoperative imaging is used to calculate the coordinates of the biopsy points and a needle insertion trajectory. Optimal trajectories are as short as possible and avoid critical vascular structures and eloquent brain regions. Possible complications include seizures, infection, and the most severe risk of intracranial hemorrhage [2]. At the biopsy point, tissue samples are extracted for histological analysis by a pathologist to classify the malignancy of the tumor for the process of determining the treatment plan for the patient. In some cases, the pathologist is not able to make a diagnosis due to the extraction of samples from nondiagnostic tissue [3]-[5]. A failure to obtain diagnostic tissue results in a repeated biopsy, prolonging the operation time and exposing the patient to additional risks.

A fiber optic probe was developed at Linköping University for simultaneous measurement of PpIX fluorescence emission and microvascular perfusion along the biopsy needle

\footnotetext{
*The study was financed by the LiU-cancer, Swedish Childhood Cancer Foundation (grant no. 2013-0043) and ALF Grants Region Östergötland (LIO-599651). *This paper is based on the material published in [1].

1,2 Michaela Maintz is with the Department of Biomedical Engineering, Linköping University, Linköping, Sweden and the Institute for Medical Engineering and Medical Informatics, FHNW, Muttenz, Switzerland michaela.maintz@students. fhnw. ch

${ }^{3}$ David Black is with the Fraunhofer MEVIS Institute for Digital Medicine in Bremen, Germany david.black@mevis.fraunhofer. de

${ }^{1}$ Neda Haj-Hosseini is with the Department of Biomedical Engineering, Linköping University, Linköping, Sweden neda.haj.hosseinilliu.se
}

insertion trajectory to improve the biopsy procedure [6] The risk of intracranial hemorrhage can be minimized by detecting blood vessels using LDF. The use of fluorescence spectroscopy (FS) in the procedure allows the detection of marked malignant tumor tissue with very high sensitivity. Fig. 1 illustrates the concept of the application and the measurements. PpIX is a photosensitizer that accumulates in brain tumor cells by administrating 5-aminolevulinic acid (5ALA) and is used to differentiate brain tumor from normal tissue. PpIX fluorescence can be excited by blue light at $405 \mathrm{~nm}$ and displays an emission peak at $635 \mathrm{~nm}$ (red). In the same spectrum, autofluorescence (AF) is collected, characterized by an increased signal intensity at around 510 $\mathrm{nm}$. When measuring the PpIX fluorescence in tissue, the major errors caused by blood interference or system error can be detected from the AF.

Biopsy procedures are followed by up to one hour of waiting for the pathological examination. Application of the optical guidance together with an optimal UI may shorten this procedure and increase its efficacy and safety. Auditory displays for medical navigation have been evaluated, resulting in benefits including improved placement accuracy, increased recognition of structure and target distance, reduced rates of clinical complication and reduced cognitive workload. For a thorough review of auditory display in image-guided interventions, see Black et al. [7]. Based on promising results of auditory display for FS measurements, the aim of the current study was to adapt the earlier auditory interface and experimentally evaluate it together with a visual UI for the aforementioned application in a modeled operating room $(\mathrm{OR})$.
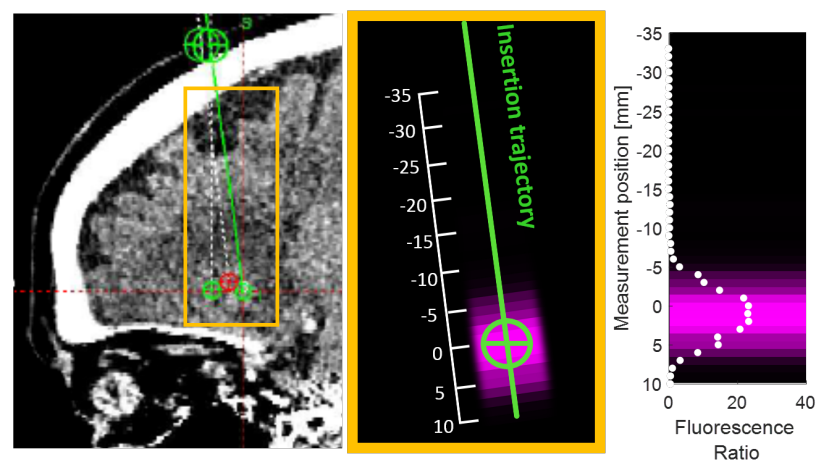

Fig. 1. A schematic of the application concept. The PpIX fluorescence ratio is zero outside of the tumor and gradually increases when approaching the tumor. The fluorescence data is re-used from [6] 


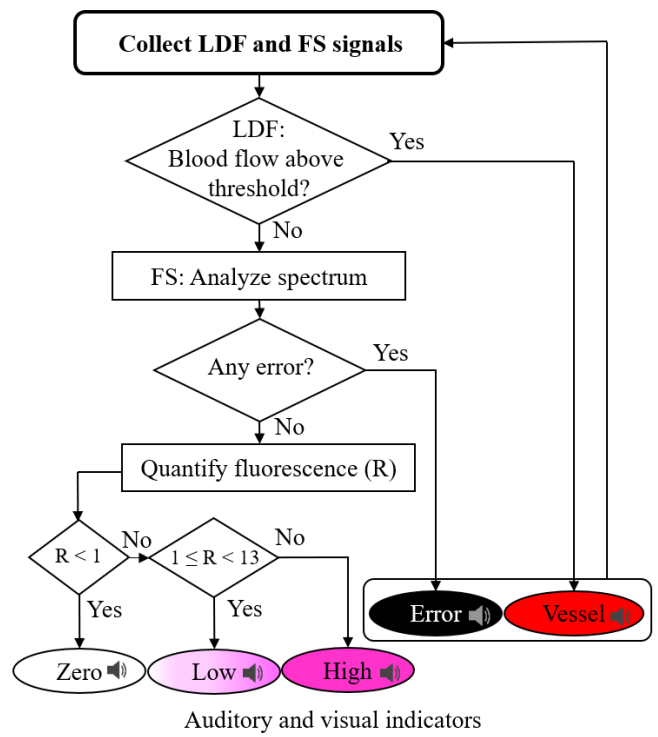

Fig. 2. The flowchart displays the principle of fluorescence spectroscopy (FS) and laser Doppler flowmetry (LDF) signal analysis in LabVIEW to generate auditory and visual indicators at each measurement site. The color of the fluorescence indicator was white, vague and intense magneta depending on the R-value. "Error" and "Vessel" were represented by black and red, respectively.

\section{Methods}

Details of the measurement setup used in the clinic are described in an earlier publication [6]. An identical experimental setup was used for performing the user response study where the hardware control and UI were developed in LabVIEW [1]. A user-centered approach was used to develop the UI features. User, OR, and safety requirements were gathered in dialogue with neurosurgeons and were integrated into the design of the UI. Thereafter, a user interaction study was conducted to test the effectiveness, efficiency and user acceptance of the UI [1].

\section{A. Experimental setup and gradient models}

A FS setup was used to replicate the measurements in the brain using a color gradient model. The system included a laser (405 nm, Zm18, Z-LASER, Germany) and a spectrometer $(580 \mathrm{~nm}$ to $1100 \mathrm{~nm}$, Avaspec-2048XL, Avantes BV, Netherlands), which were both controlled by a LabVIEW program. Measurements were performed with an integration time of $300 \mathrm{~ms}$. A fiber optic probe was connected to the FS system and the perfusion signal was simulated instead of connecting the LDF system to avoid complicated experimental perfusion measurements. The signals were registered and analyzed in real-time using LabVIEW version 2017 (National Instruments, Inc., Austin, TX, USA) and displayed by the visual and auditory interface through a loudspeaker at $50 \mathrm{~dB}$ to be at the same level of the verbal communication. As a safety measure, the laser turned off automatically when the measurement was not running. Additionally, pre-recorded sounds of medical equipment and ventilation were played back during the experiment at the same sound level that is present in the OR during brain surgery $(50 \mathrm{~dB})$.
The models were printed color gradients enclosed in cardboard boxes that produced different reflection spectra in the red optical region comparable to PpIX fluorescence. Vessel detection was replicated by a material with a distinct spectral shape and error was modelled by a material with a low fluorescence intensity at $600 \mathrm{~nm}$. The boxes had a narrow opening to allow movement of the optical probe along the color gradient. The narrow opening made the signal values reproducible in varied lighting and prevented the study participants from viewing the color gradient. In total, four sets of color gradients were produced to allow variation among the tests.

\section{B. Software design and signal analysis}

Intensities at wavelengths of $600 \mathrm{~nm}\left(I_{600 \mathrm{~nm}}\right)$ and 635 $\mathrm{nm}\left(I_{635 \mathrm{~nm}}\right)$ were extracted and scaled to communicate signals through the visual and auditory display. The signals represented the occurrence of no fluorescence, "Zero", increasing and decreasing fluorescence values, "Low", high fluorescence, "High", in case of signal blockage, "Error", and one warning signal to detect blood vessels, "Vessel". The algorithm for signal analysis is depicted in Fig. 2. If the measured signal did not produce a "Vessel" or an "Error" output, the scaled fluorescence ratio $\left(\mathrm{R}=I_{635 \mathrm{~nm}} / I_{600 \mathrm{~nm}}\right)$ for the indicators "Zero", "Low", and "High" was calculated. The thresholds were adapted from earlier clinical measurements on PpIX [8].

1) Sound design: The analyzed signals were transferred in real-time using Open Sound Control (OSC) [9] messages to trigger a specified response of the sound synthesizer created in the PureData sound programming environment [10]. The sound output depended on the R-value that was transmitted by LabVIEW. In contrast to previous work which employed a series of 4 discrete tones [11], the auditory signals in this approach had a continuous character, with a fluid transition between the tones indicating decreasing and increasing fluorescence intensity when progressing into tissue with alternating PpIX concentration. The sounds were developed to be easy to interpret and to reduce interference with other auditory devices in the operating room. Additionally, the sound was designed as a playable digital instrument' rather than a typical alarm so as not to disturb the potentially awake patient and disrupt verbal communication. The auditory and visual displays had a feedback delay equal to the integration time of the spectrometer. Sound was played back immediately after the value was sent to the sound synthesizer. The tones were designed to be the same length as the integration time in order to receive an uninterrupted auditory signal. For increasing or decreasing low fluorescence values $(1 \leq R<13)$, a variation of tones was played, which represented the continuous transition of fluorescence values. For low signals, three simultaneous oscillators were generated at 277, 370 and $466 \mathrm{~Hz}$. With an increasing Rvalue, frequency modulation took place. Starting at $R=1$, the modulating frequency linearly increased from $2 \mathrm{~Hz}$ to $15 \mathrm{~Hz}$, until the R-value approached 13. As R decreased, so did the modulating frequency, indicating a decrease of fluorescence 


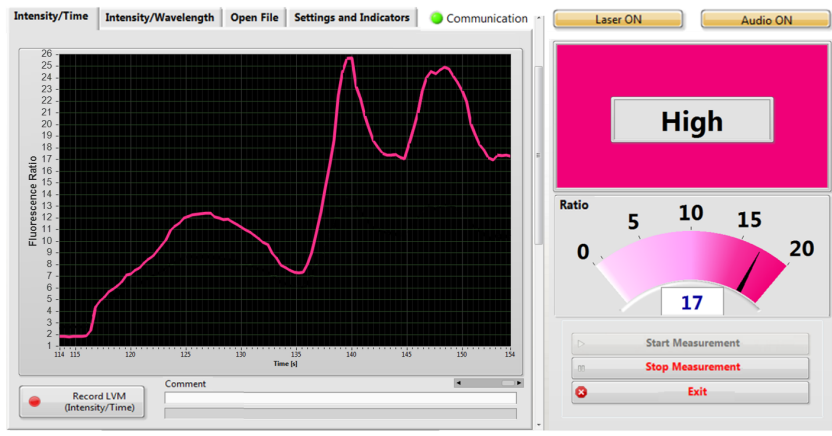

Fig. 3. The visual user interface indicating a high fluorescence R-value during measurement.

which was still below the "High" threshold. Crossing above the R-value 13 signals the maximum "High" intensity value. This was transmitted by a jump in the frequencies of the three oscillators from the initial frequencies $(277,370$, and $466 \mathrm{~Hz})$ to an octave higher $(554,740$, and $940 \mathrm{~Hz}$ ). For error feedback, a unique tone was created that contrasted both the recorded OR sounds as well as the aforementioned continuous fluorescence feedback tones. For a detailed description, see [11]. For vessel feedback, a tone similar to a heartbeat was employed.

2) Visual design: The visual interface was split vertically into two windows, with detailed measurement values and settings in the left window and measurement controls and signal indicators located in the right window. The left window included four tabs, where the first tab visualized intensity of selected wavelengths vs. time and the second tab showed the whole measured spectrum. The previous measurements could be opened and viewed in the third tab. In the fourth tab, detailed measurement settings could be adjusted and indicator thresholds could be viewed. Large color and text indicators displayed different measurement values, while the scale indicated the R-value that represented the measured optical value. The signal values were each depicted by a specific color in a color box surrounding the text indicator. The audio ON/OFF switch in the UI allowed the activation and deactivation of the auditory output. Fig. 3 shows the visual interface representing the indicator for "High" fluorescence in the first tab. The left side of the window shows the variation of fluorescence along a color gradient model.

\section{Study participants}

The volunteer subjects $(n=15)$ taking part in the study had backgrounds in engineering and natural sciences ( $\mathrm{n}=$ 14) and in humanistic studies $(n=1)$. The participants were 23 - 51 years old. None of the subjects declared any vision or hearing impairment. No ethical approval was required for this type of study. The participants were informed of their rights and gave written informed consent prior to the tests.

\section{User interaction and acceptance study}

The user interaction study was performed in an environment that replicated the OR, where the user interaction with the interface was recorded and evaluated. Specifically, the ability to perform the measurements and distinguish the warning signals at the same time was tested with respect to accuracy. This employed color gradient models with different signal sequences unknown to the participants. The participants were informed of the clinical application and of the UI evaluation procedure and were instructed to consider the circumstances in the OR and react as quickly as possible. In Test 1 , the user's ability to identify the auditory signals by intuition was evaluated. After a brief training, the response accuracy to the auditory signals only (Test 2) and to both of the auditory and visual signals under the influence of background noise was tested (Test 3). Throughout Test 3, the interaction of the user with the visual interface was recorded. A memory test (Test 4) was conducted 7-10 days after the participants' $(n=11)$ last usage of the UI. The usability of the UI was evaluated with the common Van der Laan user acceptance scale [12] to determine the usefulness of the UI and user satisfaction. For this, a 5point Likert scale questionnaire with 9 items representing either practicability (usefulness) or likability (satisfaction) was used. Additionally, the test participants' perception of the difficulty of use and satisfaction with the auditory and visual interface was polled.

\section{RESUlTS}

\section{A. User interaction study}

The mean response accuracy of the test subjects was over $91 \%$ in all tests. Table I displays the response accuracy of all participants to the individual signal indicators throughout the total occurrences of the indicator in the entire experiment. Fields with no values are instances that did not occur and can also be described as 0\%. The percentages in bold are the correct responses. "Error" was sometimes mistaken for "Zero" (7\%) and "Low" (7\%). In 16\% of all instances, "Zero" was mistaken for "Low". Throughout Test 3, an average of four participants viewed the visual interface, whereas the rest $(\mathrm{n}=11)$ exclusively relied on the auditory display. $87 \%$ of the test subjects stated that they did not have difficulties in distinguishing the auditory signal feedback from the recorded OR noises, whereas the remaining 13\% stated that the background noise might have influenced their response accuracy.

TABLE I

CONFUSION MATRIX DISPLAYING THE PERCEIVED SIGNAL VALUES WHEN A SPECIFIC SIGNAL VALUE WAS PLAYED IN ALL TESTS

\begin{tabular}{|c|c|c|c|c|c|c|}
\hline & \multicolumn{5}{|c|}{ Played signal indicator } \\
\hline & & Error & Zero & Low & High & Vessel \\
\hline \multirow{5}{*}{$\begin{array}{l}\text { Perceived } \\
\text { signal } \\
\text { indicator }\end{array}$} & Error & $84 \%$ & & & $6 \%$ & \\
\hline & Zero & $7 \%$ & $84 \%$ & & & \\
\hline & Low & $7 \%$ & $16 \%$ & $99 \%$ & & \\
\hline & High & $2 \%$ & & $1 \%$ & $93 \%$ & \\
\hline & Vessel & & & & $1 \%$ & $100 \%$ \\
\hline \multicolumn{2}{|c|}{ Total occurrence } & 56 & 71 & 251 & 101 & 56 \\
\hline
\end{tabular}




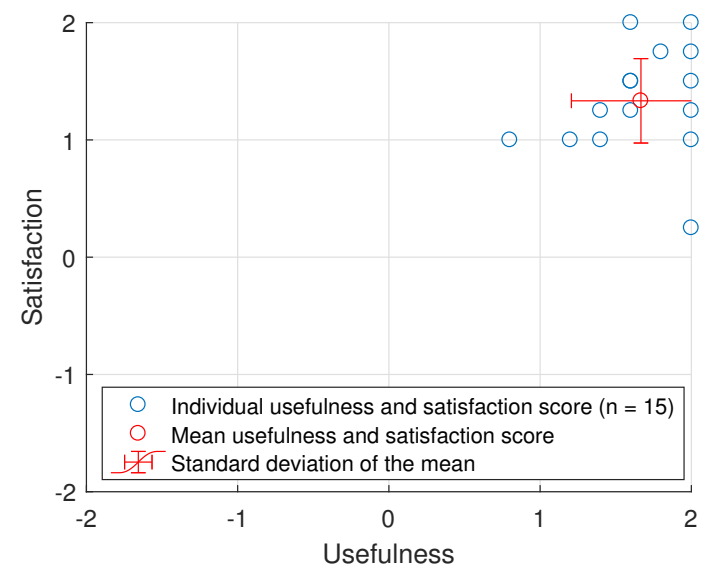

Fig. 4. Usefulness and satisfaction scores of the user interface derived from the user acceptance evaluation.

\section{B. User acceptance}

The test subjects' individual perception of their satisfaction with, and the usefulness of the UI is shown in Fig. 4. On a scale between -2 (strongly disagree) and 2 (strongly agree), the average UI usefulness had a score of $1.7 \pm 0.4$ and user satisfaction had an average score of $1.4 \pm 0.5$. In the evaluation of the feedback speed of $300 \mathrm{~ms}$ per signal, none of the participants expressed any dissatisfaction. All the test subjects reported that the auditory and visual interface was easy to understand.

\section{DISCUSSION}

During stereotactic brain tumor biopsies, the multimodal approach of delivering real-time measurement feedback to the neurosurgeon can increase the efficiency and accuracy of data communication in the procedure. Simple, unambiguous, and large visual indicators allow the user to act fast when confronted with a critical situation. The colors representing the signals, especially magenta for "High" PpIX fluorescence and red for "Vessel" detection allow quick recognition as they can be easily associated with the assigned function. The text indicators eliminate the need to interpret the data. The scale gives detailed information of the exact measured value. The organization of measurement values and settings allows a non-cluttered display of data with reduced visual complexity.

The user interaction study was conducted to investigate the users' ability to interpret the auditory signals and to determine the usefulness and user satisfaction of the UI. With response accuracies of over $91 \%$ in the four conducted tests, examining intuition, accuracy, and memory, it can be said that the signal feedback is highly intuitive, easy to remember, and evokes accurate responses. The fact that a majority of test participants (11 out of 15) chose not to view the visual interface throughout Test 3 indicates that the users were comfortable with determining the signal indicators using only auditory cues. The "Low" and "Vessel" signal indicators were easily recognizable with high response accuracies
(Table I), whereas conveying "Error" and "Zero" with an accuracy of $84 \%$ could be improved. Throughout all tests, the participants were able to precisely determine increasing and decreasing "Low" values, which in fluorescence-guided biopsies is important when anticipating tumor appearance in a trajectory. The results of the user acceptance study showed that the UI was well received by all test participants.

In the future, following validation, the degree to which the UI is beneficial to medical staff during biopsies in the OR can be evaluated. Fluorescence measurement values can be combined with intraoperative navigation systems to visualize fluorescence occurrence along the biopsy trajectory. This could give an approximation of tumor location after a possible brain shift. This UI could also be applied for use in open-brain tumor resections.

\section{ACKNOWLEDGMENT}

The authors would like to thank Karin Wårdell, Prof., Johan Richter and Peter Milos, Neurosurgeons at the Department of Neurosurgery, Linköping University Hospital for the initial evaluation of the system.

\section{REFERENCES}

[1] M. Maintz, "Data representation for fluorescence guided stereotactic brain tumor biopsies: Development and evaluation of a visual and auditory user interface," Bachelor Thesis, Inst. for Medical and Analytical Technologies, Univ. of Applied Sciences and Arts Northwestern Switzerland, Switzerland and Dept. of Biomedical Engineering, Linköping Univ., Sweden, 2018.

[2] M. Field, T. F. Witham, J. C. Flickinger, D. Kondziolka, and L. Dade Lunsford, "Comprehensive assessment of hemorrhage risks and outcomes after stereotactic brain biopsy," J of Neurosurgery 94, 545$551,2001$.

[3] J. D. Waters, D. D. Gonda, H. Reddy, E. M. Kasper, P. C. Warnke, and C. C. Chen, "Diagnostic yield of stereotactic needle-biopsies of sub-cubic centimeter intracranial lesions," Surgical Neurology International 4, S176-S181, 2013.

[4] C.-C. Chen et al., "Stereotactic brain biopsy: Single center retrospective analysis of complications," Clinical neurology and neurosurgery, vol. 111 (10), 835-839, 2009.

[5] F. V. Aker, T. Hakan, S. Karadereler and M. Erkan, "Accuracy and diagnostic yield of stereotactic biopsy in the diagnosis of brain masses: comparison of results of biopsy and resected surgical specimens," Neuropathology 25, 207-213, 2005.

[6] N. Haj-Hosseini, J.C.O. Richter, P. Milos, M. Hallbeck, K. Wårdell, "5-ALA fluorescence and laser Doppler flowmetry for guidance in a stereotactic brain tumor biopsy," Biomedical Optics Express, 9 (5), 2284-2296, 2018.

[7] D. Black, C. Hansen, A. Nabavi, R. Kikinis, H. Hahn, "A Survey of Auditory Display in Image-Guided Interventions," J. Computer Assisted Radiology and Surgery 12 (10), 1665-1676, 2017.

[8] J. Richter, N. Haj-Hosseini, M. Hallbeck, and K. Wårdell, "Combination of Hand-Held Probe and Microscopy for Fluorescence Guided Surgery in the Brain Tumor Marginal Zone, Photodiagnosis and Photodynamic Therapy 18, 185-192, 2017.

[9] M. Wright, "Open Sound Control: an enabling technology for musical networking," Org. Sound, vol. 10 (3), 193-200, 2005.

[10] M. Puckette, "Pure Data: another integrated computer music environment," in Second Intercollege Computer Music Concerts, 37-41, 1996.

[11] D. Black, H. K. Hahn, R. Kikinis, K. Wårdell, and N. Haj-Hosseini, "Auditory display for fluorescence-guided open brain tumor surgery," International Journal of Computer Assisted Radiology and Surgery 13(1), 25-35, 2018.

[12] J. D. Van Der Laan, A. Heino, and D. De Waard, "A simple procedure for the assessment of acceptance of advanced transport telematics," Transportation research. Part C, Emerging technologies, 5 (1), 1-10, 1997. 